\title{
GvHD Lung Symptom Score 3
}

National Cancer Institute

\section{Source}

National Cancer Institute. GvHD Lung Symptom Score 3. NCI Thesaurus. Code C131053.

Severe symptoms (shortness of breath at rest; requiring $\mathrm{O} 2$ ). 